\title{
Las experiencias de trabajo de campo para aprender y enseñar geografía: El caso de la comunidad educativa de Osorno, Chile
}

\author{
Gladys Moreno Schmidt ${ }^{1}$ \\ María Luisa Zagalaz Sánchez ${ }^{2}$ \\ Luisa Magaly Elzel Castro ${ }^{3}$
}

Recibido: 21 de marzo de 2018 - Aprobado: 23 de abril de 2018

\begin{abstract}
Resumen
Se presenta un estudio práctico sobre el aprendizaje de la geografía en la comunidad educativa de nivel secundario en la ciudad de Osorno (Chile) aplicando el trabajo de campo como una enseñanza esencialmente activa que abarca el conocimiento del entorno y el aprendizaje por descubrimiento. El objetivo del trabajo examina la relación entre prácticas pedagógicas centradas en el aula y las usadas en experiencias propias del trabajo de campo con actividades al aire libre en el ámbito local. La investigación responde a un estudio empírico-descriptivo, en el que se aplican instrumentos a profesores de establecimientos educacionales y estudiantes de octavo semestre de Pedagogía en Historia y Geografía de la Universidad de Los Lagos. Se concluye que el trabajo de campo tiene valor en tanto la actividad tenga relación con la problemática y el análisis de lo observado y no solo en la descripción de los elementos.
\end{abstract}

Palabras clave: Educación, aprendizaje activo, enseñanza de la geografía, trabajo de campo

Chilena. Magíster en Pedagogía y Gestión Universitaria, Universidad Metropolitana de Ciencias de la Educación, Chile. Académica Universidad de Los Lagos, Osorno, Chile. E-mail: gmoreno@ulagos.cl

2 Española. Doctora en Psicopedagogía, Universidad de Jaén, España. Académica, Universidad de Jaén, España. E-mail: Izagalaz@ujaen.es

3 Chilena. Doctora en Aspectos Educativos y Socioculturales de la Actividad Física y el Deporte, Universidad de Jaén, España. Académica, Universidad de Los Lagos, Osorno, Chile. E-mail: lelzel@ulagos.cl 


\title{
Experiences of field work to learn and teach geography: The case of an educational community of Osorno, Chile
}

\begin{abstract}
The article presents a practical case about the study of geography in the secondary educational level at the city of Osorno, Chile, applying the fieldwork as an essential active education encompassing the environment knowledge and learning by discovering. The objective is to review the relation among classroom-focused pedagogical practices and the use of fieldwork experiences with local and outdoor activities. The research responds to an empiricaldescriptive study applying tools to teachers from schools in Osorno, and to students of the Eighth Semester of History and Geography Pedagogy from Universidad de Los Lagos. It finds that the fieldwork has value provided the activity is related to the issue and observations analysis and not only in the description of elements.
\end{abstract}

Keywords: Education, active learning, geography teaching, work field

\section{As experiências de trabalho de campo para aprender e ensinar geografia: 0 caso da comunidade educativa de Osorno, Chile}

\section{Resumo}

Apresenta-se um estudo prático sobre o estudo da geografia na comunidade educativa de nível secundário na cidade de Osorno (Chile) aplicando o trabalho de campo como uma forma de ensino essencialmente ativa que abrange 0 conhecimento do entorno e aprendizado através da descoberta. 0 objetivo do trabalho examina a relação entre práticas pedagógicas centradas na sala de aula e as que são usadas em experiências próprias do trabalho de campo com atividades ao ar livre no âmbito local. A pesquisa responde a um estudo empírico-descritivo, no que se aplicam instrumentos a professores de estabelecimentos educacionais e a estudantes de oitavo semestre de Pedagogia em História e Geografia da Universidade de Los Lagos. Conclui-se que o trabalho de campo tem valor desde que a atividade tenha relação com a problemática e a análise do observado e não só na descrição dos elementos.

Palavras-chave: Educação, aprendizado ativo, ensino da geografia, trabalho de campo

\section{Argumentación teórica y fundamentación del problema}

La Reforma Educacional chilena está fundamentada en la necesidad de instituir acciones destinadas a mejorar los procesos educativos sobre la base de tres principios fundamentales: equidad, calidad y participación (Ministerio de Educación, 1997). El principio de calidad proporciona contexto al presente trabajo, considerando que establece la adopción de estilos pedagógicos 
centrados en el aprendizaje activo de los estudiantes dentro y fuera del aula. Lo anterior se considera extensible hacia la enseñanza de la geografía y, en consecuencia, también hacia las actividades de aprendizaje planificadas en contacto con el entorno escolar y el espacio geográfico.

Implementar el principio anterior significa disponer de un docente familiarizado permanentemente con los fundamentos disciplinarios de la geografía y capacidad creativa para apoyar al estudiante en la construcción de nuevos conocimientos. Las situaciones de aprendizaje constituyen una de las prioridades establecidas en la Reforma Educacional chilena que, a su vez, debiera tener un impacto importante en aquellas universidades formadoras de profesores/as y su inherente función investigativa en educación.

Desde el ámbito conceptual y teórico se reconoce la relevancia singular del trabajo de campo como aporte disciplinario propio de la ciencia geográfica en el ámbito de la enseñanza-aprendizaje de la geografía. Simultáneo al análisis del espacio, el trabajo de campo constituye una estrategia didáctica para producir experiencia de aprendizaje activo mediante la observación y el estudio de la realidad del entorno inmediato. Sin embargo, no se ha estudiado de manera sistemática en nuestro país el nivel de impacto que, eventualmente, esta actividad haya logrado en nuestras comunidades educativas en el nivel de la enseñanza media. La necesidad de resolver dicho problema motiva este artículo, que determina la importancia del trabajo de campo como metodología activa en el aprendizaje de la geografía y el análisis de la realidad extraaula en la comunidad educativa de la ciudad de Osorno, Chile.

A continuación se exponen los fundamentos teóricos relativos al problema antes señalado. La enseñanza de la geografía, al inicio de la década de 1990, mantenía la influencia heredada de la geografía descriptiva, consolidándose como la base de una enseñanza tradicional (Durán et al., 1993). Se consideraba que dicha enseñanza ya estaba siendo desplazada por una geografía basada en un aprendizaje más activo, entendiendo por ello el estudio del entorno y la enseñanza por descubrimiento, lo que potenciaba el saber territorial innato que toda persona posee. Esto fue considerado como una evolución de la ciencia geográfica, desde una descripción y explicación de los paisajes hacia una relación hombre-medio.

Comparando el modelo de enseñanza denominado "paradigma de la geografía descriptiva y explicativa" frente a las nuevas orientaciones pedagógicas que acompañan al proceso reformador, se evidencia la necesidad de modificar la metodología hasta ahora utilizada, que se basa en la transmisión de conocimientos que conducen a un aprendizaje esencialmente mecánico y repetitivo. El cambio pedagógico se realizará en la medida en que los contenidos surjan de la dinámica social en la que profesor y estudiante son protagonistas de los 
actos sociales y escolares, asumiendo la realidad como objeto de estudio en el diario vivir e interactuando con el medio que lo rodea (Santiago, 2008).

Los cambios que se pretende realizar en la enseñanza de la geografía responden a la existencia de nuevos escenarios, donde se encuentra una sociedad inmersa en el proceso de globalización, conocimiento, tecnología avanzada, en un mundo virtual cada vez más cercano a la población escolar y universitaria (Araya y González, 2009).

En la actualidad, los expertos que estudian las temáticas de la enseñanza geográfica persisten en el cuestionamiento de la labor formativa, obsoleta y envejecida del aula, significando con ello que todavía se evidencia el apego a la visión centrada en transmitir contenidos de aspectos disciplinares de esta ciencia, fijando en la mente de los estudiantes solo memorización como parte de su aprendizaje (Santiago, 2017). La confluencia de los procesos de observación y descripción geográfica, mediante observaciones del entorno inmediato, permiten la lectura del espacio, ya que el estudiante entiende el funcionamiento de éste a través del contacto con sus formas de organización, para reflexionar posteriormente sobre su entorno y la resolución de problemas (Pérez, 2006).

La enseñanza de la geografía no puede quedar al margen de las innovaciones metodológicas, sobre todo en lo que respecta a la búsqueda de una enseñanza más activa, entendiendo por ello el estudio del entorno y la enseñanza por descubrimiento. Para ello se enfatiza el conocimiento espacial innato existente en cada estudiante, importando la enseñanza del entorno, del medio cercano, para que el discente sea capaz de sistematizar su conducta respecto del mismo. La enseñanza por descubrimiento debe lograr que los estudiantes se encuentren cómodos en la ambigüedad y contradicción permanente de todo genuino conocimiento, satisfechos intelectualmente al estudiar y comprender por sus propios medios la insatisfacción que caracteriza el mundo de nuestros días (Sánchez, 1997).

Desde la perspectiva específica del profesorado, se establece que en los nuevos contenidos curriculares y en la didáctica se planteen metodologías desde un enfoque realista y basadas en problemas (Tatter, 1983; Araya y González, 2009). Considerando el constructivismo en la enseñanza de las ciencias sociales, se plantea de forma paralela que se deben tomar en cuenta las experiencias vividas por los alumnos y alumnas y su subjetividad, agregando que este conocimiento no es producto de una interacción únicamente personal, sino que requiere de interacciones constantes entre sujeto y medio y, por lo tanto, el uso de metodologías activas en la enseñanza de la geografía son fundamentales (Souto, 1999; Rodríguez, 2007). Las actividades constructivistas no constituyen una tarea individual sino interpersonal, en la que el estudiante interactúa con el profesor, con los compañeros y con la comunidad local. 
El análisis de la importancia del trabajo de campo como estrategia didáctica y el conocimiento del espacio geográfico, partiendo desde momentos históricos pasados hasta llegar al presente, en donde

"las excursiones pedagógicas y trabajos de campo han sido desde los inicios de la enseñanza de la geografía estrategias didácticas valiosas, y a pesar del inmenso avance de la ciencia y la tecnología actual, que ha introducido cambios significativos en los métodos de estudio de muchas disciplinas, este tipo de actividades tiene en esta ciencia más vigencia que nunca, por cuanto es la mejor manera de verificar en el terreno lo que teóricamente se expone en las aulas de clase". (Montilla, 2005:108)

Las metodologías para la enseñanza de la geografía han de basarse en la observación y en la experiencia, pero una observación que resulte analítica, es decir, que pretenda explicar la causalidad de los hechos, en la que el profesor tenga una actitud dinámica, de tal forma que los estudiantes puedan tener un conocimiento profundo de los conceptos geográficos (Tatter, 1983). Para ello se plantea el trabajo de campo como una forma de mostrar evidencias sobre la condición natural del ambiente y examinar los problemas que resulten de la interacción del hombre con su medio.

Otra perspectiva de la importancia del trabajo de campo en la enseñanza de la geografía se relaciona con la forma ideal de interpretar realmente lo que ocurre en el ambiente, para que los estudiantes se percaten de la frecuencia de los cambios y la fragilidad del ecosistema. Para atender esta diversidad de percepción se puede hacer uso de recursos, por ejemplo, mapas, fotografías, receptores de GPS, estaciones meteorológicas, entre otros (Montilla, 2005). Por lo tanto, el trabajo de campo tiene por objeto que el estudiante finalice su aprendizaje conceptualizando a partir de lo observado y no repitiendo la definición del texto, como consecuencia de la dificultad de abstracción, formulando conceptos científicos a partir de lo perceptual que a su vez se organiza en sistemas conceptuales (Giacobbe, 1998).

El trabajo de campo requiere sus propias destrezas, para lo cual se ha desarrollado un gran número de técnicas apropiadas a este tipo de trabajos, en el que el profesor, al utilizar esta metodología, requiere de habilidades concretas: aptitud de enseñar en campo abierto, planificar y organizar a su grupo de estudiantes al aire libre (Bailey, 1983).

En el presente estudio, referido a la comunidad educativa de la ciudad de Osorno, se plantean los siguientes objetivos:

- Determinar el nivel de importancia del trabajo de campo en el aprendizaje de la geografía y análisis de la realidad extra aula. 
- Establecer la percepción que, desde sus propias experiencias, tienen docentes y estudiantes (pedagogos en formación) acerca del trabajo de campo.

\section{Método}

Este estudio responde a un enfoque mixto, en el que se realiza un análisis cuantitativo y cualitativo, y tiene un diseño de tipo empírico-descriptivo que busca observar los fenómenos que constituyen la realidad del ambiente geográfico y sus interrelaciones (Hernández et al., 1998).

Para esta investigación se elaboraron dos instrumentos: cuestionario y entrevista.

La perspectiva teórica se realizó mediante la técnica de juicio de experto con la participación de académicos de la Universidad de La Serena, Chile; Universidad Pedagógica Experimental Libertador, Venezuela, y Universidad de Los Lagos, Chile.

Para interactuar con los sujetos de estudio, en primer lugar se aplicó el cuestionario (dieciséis preguntas) a 62 profesores de enseñanza secundaria en la especialidad de Historia y Geografía que laboran en liceos municipales, colegios particulares subvencionados y particulares pagados de la ciudad de Osorno. En el ámbito universitario, se incorporó a treinta estudiantes pertenecientes al octavo semestre de la carrera de Pedagogía en Historia y Geografía de la Universidad de Los Lagos, Osorno. Posteriormente, se realizó el análisis de calidad de los ítems. Para ello se calculó la varianza como estadístico descriptivo para cada uno de los ítems del cuestionario. Se determinó la fiabilidad de la elección de los ítems mediante el índice Alfa de Cronbach, que refleja la homogeneidad de los ítems, es decir, el grado en que todos los ítems hacen referencia al constructo que se pretende medir con el cuestionario, así como su calidad técnica.

El segundo instrumento, la entrevista, fue respondida por quince profesores y ocho estudiantes, la muestra corresponde a una selección de los participantes del primer instrumento.

A continuación se especifican características principales de ambos instrumentos.

\section{a) Cuestionario}

La elaboración del cuestionario se centró en indicadores de información relevante sobre el trabajo de campo como estrategia metodológica para enseñar y aprender geografía. 
Se utilizó la escala Likert para medir la actitud con respecto a situaciones de orden metodológico. Para un mejor análisis y de acuerdo al sentido de las preguntas, estas fueron clasificadas en dos categorías:

1. Importancia del trabajo de campo.

2. Observación directa.

\section{b) Entrevista}

La finalidad de aplicar este segundo instrumento fue profundizar en la opinión de los profesores y estudiantes incorporados al estudio, cuyo enfoque es caracterizar las actitudes frente a experiencias reales tenidas en el contacto con la realidad extra-aula. Este instrumento de indagación permitió complementar el nivel de certeza obtenida mediante el cuestionario basado en la escala Likert.

Las cinco preguntas abiertas, que fueron respondidas por escrito en términos de percepción de los entrevistados, tuvieron el propósito de complementar las respuestas del primer instrumento. En una primera etapa de análisis se realizó una lectura de las entrevistas, apoyada en una transcripción textual de cada una de las respuestas, tanto por docentes como por estudiantes de pedagogía, acerca de sus percepciones y prácticas relativas al trabajo de campo.

\section{Resultados}

\section{a) Cuestionario}

Los ejes centrales de este estudio están orientados a la utilización de la observación directa como estrategia metodológica, el trabajo de campo y su impacto respecto a la motivación, desarrollo de valores, actitud crítica, sistematización de los contenidos, logro de aprendizajes significativos y como metodología didáctica.

Una vez obtenidas las respuestas del cuestionario, se codificó la información para su posterior procesamiento, se elaboró una matriz de datos y éstos se trasvasijaron al software estadístico SPSS 20.0, el cual permite obtener tanto las frecuencias como los porcentajes. Posteriormente, dicha información fue exportada a Excel, lo que permite representar gráficamente toda la información, para finalmente hacer un comentario de los gráficos obtenidos. 
A continuación se presenta un análisis selectivo -apoyado en figuras- referido a las dos categorías, importancia del trabajo de campo y observación directa, y las interrogantes respectivas clasificadas.

\section{Categorías de clasificación}

Tabla 1

Síntesis de interrogantes según categorías

\begin{tabular}{|l|l|}
\hline \multicolumn{1}{|c|}{ CATEGORÍAS } & \multicolumn{1}{|c|}{ N $^{0}$ DE LA PREGUNTA } \\
\hline I. Importancia del trabajo de campo & $1,2,3,5,6,10,12,13,16$ \\
\hline II. Observación directa & $4,7,8,9,11,14,15$ \\
\hline
\end{tabular}

Fuente: Elaboración propia

Se inicia el análisis con la categoría: Importancia del trabajo de campo.

\section{Categoría: Importancia del trabajo de campo}

\section{Figura 1}

Pregunta 1: ¿Usa metodologías de observación directa para enseñar Geografía?

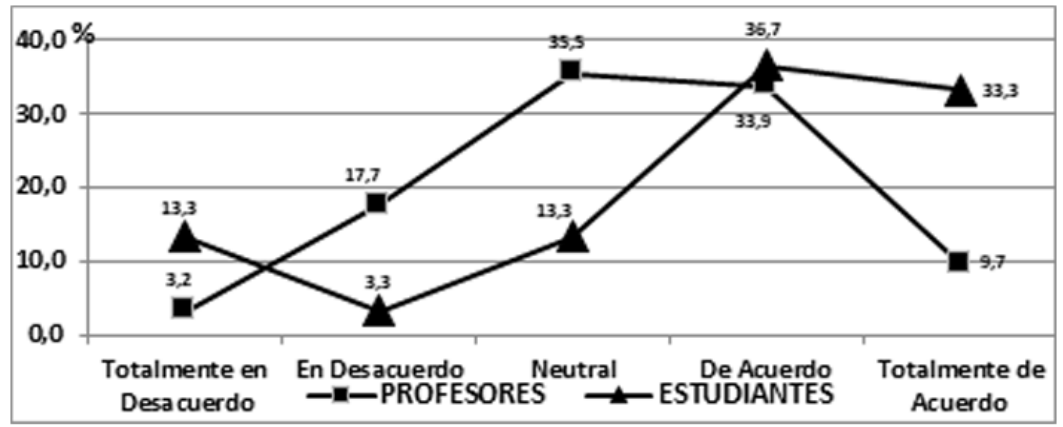

Fuente: Elaboración propia

En su conjunto, las actitudes muestran fuertes diferencias porcentualmente expresadas, a través de las cuales los estudiantes opinan positivamente; con una leve excepción en De Acuerdo. Es oportuno establecer que los docentes deben enfrentar dificultades en sus establecimientos al momento de decidir una actividad de terreno con sus alumnos; situación que resulta coherente con lo mostrado en la figura 1. 


\section{Figura 2}

Pregunta 2: Un trabajo de campo planificado, ¿favorece la creación de un sujeto consciente de su realidad?

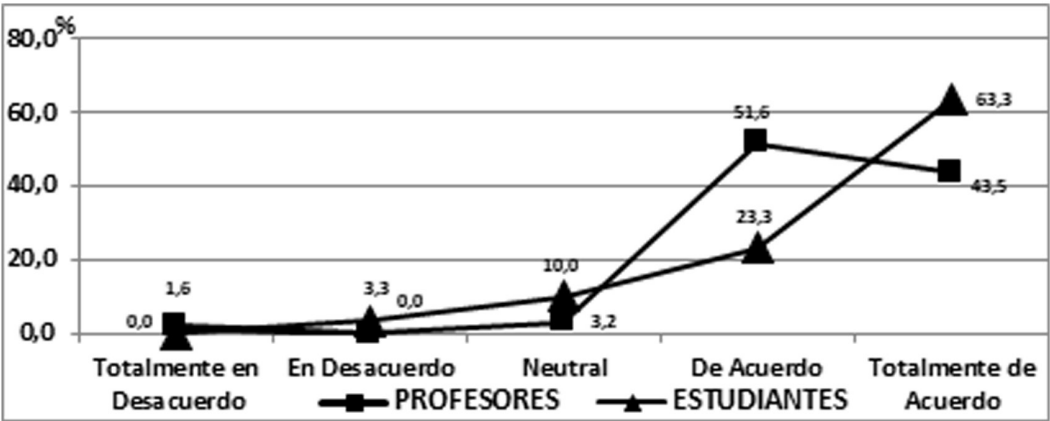

Fuente: Elaboración propia

Profesores y estudiantes están Totalmente de Acuerdo, es decir, suscriben que un trabajo de terreno previamente orientado hace posible el logro de objetivos de mayor compromiso, por tener un carácter valórico. En este caso, puede inscribirse la dinámica de los espacios en términos de deterioro o conservación de los ambientes naturales.

\section{Figura 3}

Pregunta 3: La utilización del trabajo de campo,

¿ayuda a la comprensión de procesos que ocurren sobre el espacio geográfico?

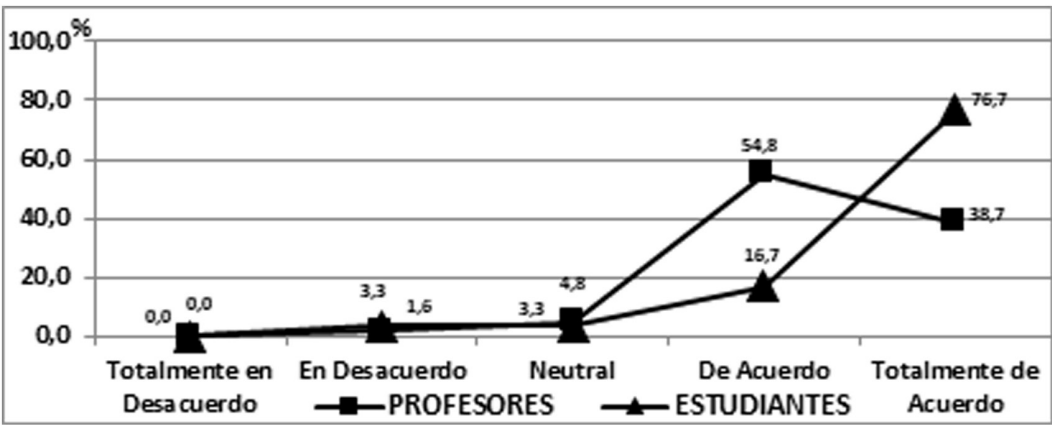

Fuente: Elaboración propia

Tanto profesores como estudiantes concuerdan en ratificar certeza del trabajo de campo respecto del análisis espacial. Ello puede significar una buena base para implementar programas de perfeccionamiento para prácticas en terreno. 
Figura 4

Pregunta 5: El trabajo de campo, ¿favorece el desarrollo de una actitud crítica del estudiante sobre el entorno?

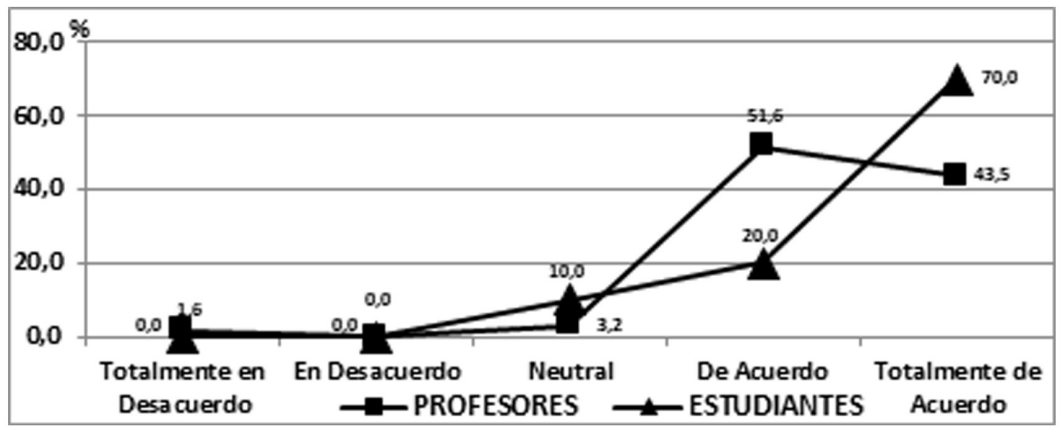

Fuente: Elaboración propia

Tanto estudiantes como profesores coinciden en su acuerdo en cuanto a favorecer la formación de una actitud crítica frente a nuestro problema planetario.

Se insiste y reitera, en el contexto de comparar entre los dos actores, que los estudiantes -como en otras respuestas anteriores- se concentran mayoritariamente en la opción Totalmente de Acuerdo (70\%); mientras que los profesores, más cautelosos, solo se manifiestan en un 43,5\%.

Figura 5

Pregunta 6: El contacto directo con la realidad, ¿favorece el desarrollo de valores en los estudiantes?

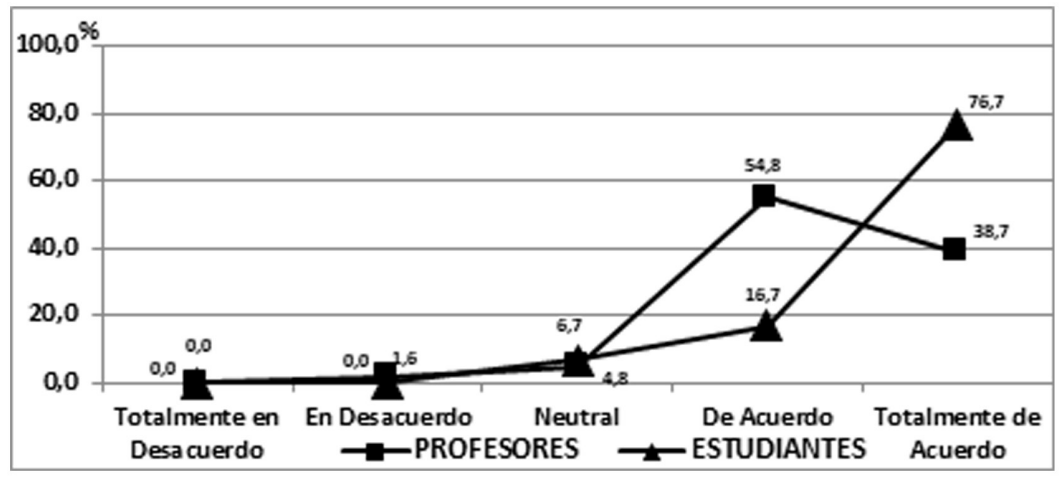

Fuente: Elaboración propia

La comparación posible se sitúa en el ámbito De Acuerdo: ambos grupos encuestados superan un $90 \%$ según acuerdos acumulados. No obstante que, 
para Totalmente de Acuerdo, los alumnos alcanzan un $76,7 \%$. Al mismo tiempo, los profesores alcanzan 38,7\%. Se puede decir que los profesores, por experiencia, aplican más realismo a sus respuestas, en cambio los estudiantes están en la posición de alumnos universitarios en proceso de formación con una mirada más positiva.

\section{Figura 6}

Pregunta 10: La aplicación de metodologías de observación directa, ¿evidencia aprendizajes significativos?

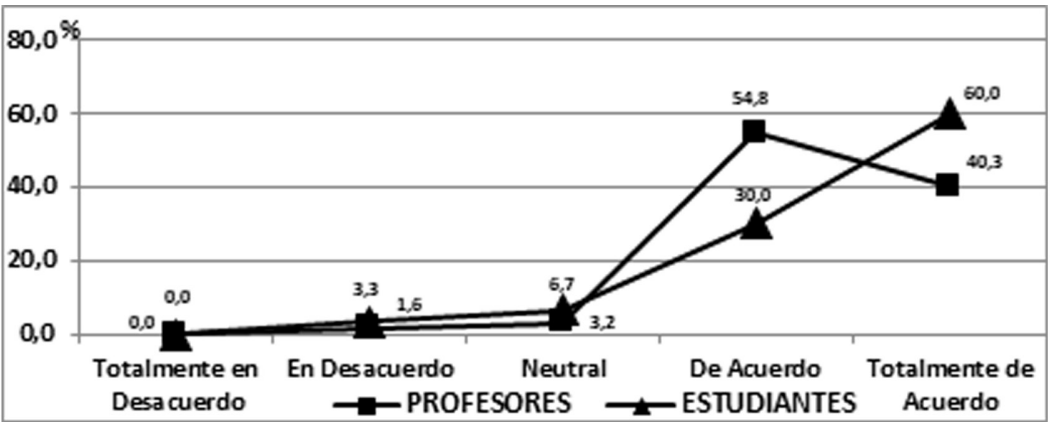

Fuente: Elaboración propia

En esta oportunidad nuevamente profesores y estudiantes coinciden en sus respuestas, manifestando su acuerdo (acumulado) el 95,1\% de los docentes y el $90 \%$ de los estudiantes, en cuanto a condicionar aprendizajes significativos en el contexto de metodologías de observación, lo que constituye un escenario positivo para incrementar experiencias tanto en la formación de docentes (prácticas profesionales) como en la docencia de los profesores activos.

Figura 7

Pregunta 12: El objetivo de las salidas a terreno, ¿permite potenciar el aprendizaje de los estudiantes?

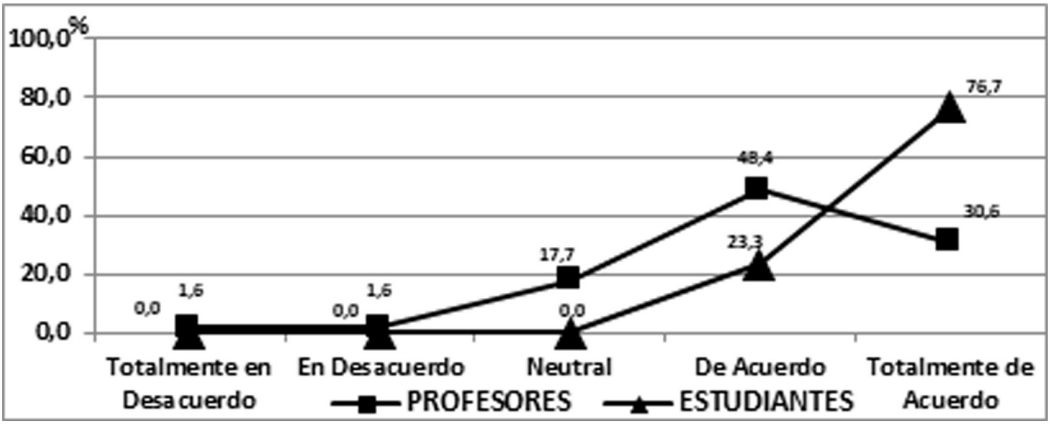

Fuente: Elaboración propia 
Estudiantes y profesores resuelven con similar intensidad su aprobación al problema planteado. Los 20 puntos porcentuales que sitúan a los docentes por debajo del porcentaje estudiantil 79\% respecto de 100\%, (diferencia entre De Acuerdo y Totalmente de Acuerdo de profesores y estudiantes) pudieran reflejar el impacto de las limitaciones del ejercicio docente en los establecimientos educacionales de la ciudad.

Figura 8

Pregunta 13: ¿Desarrolla sistemáticamente el trabajo de campo como metodología para la enseñanza de la geografía?

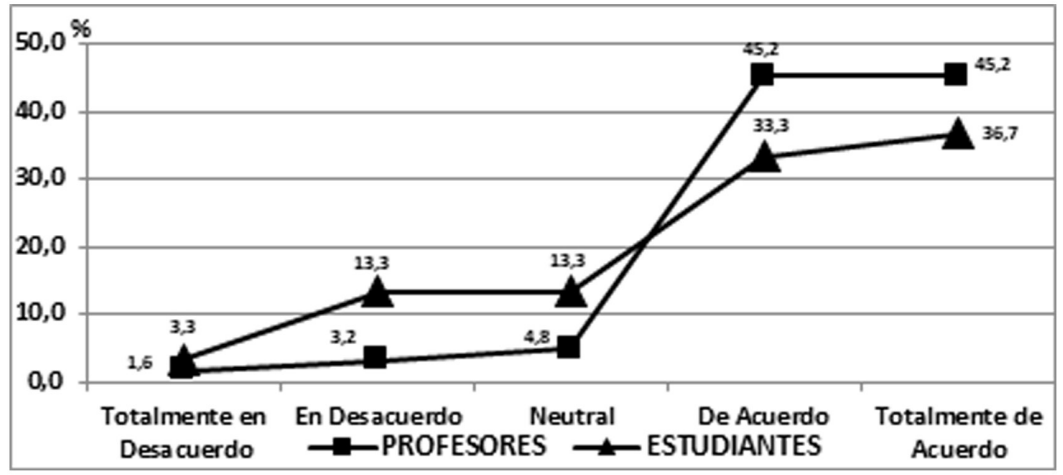

Fuente: Elaboración propia

Los estudiantes muestran un 33,3\% y los profesores un $45,2 \%$ en cuanto a los acuerdos, la diferencia entre ambos es de un 11,9\%. Además, esta situación impacta en los porcentajes que corresponden a los En Desacuerdo y Neutral de los alumnos, que totalizan un 29,9\%; mientras que en los docentes el porcentaje es de 9,6\%.

Figura 9

Pregunta 16: El trabajo de campo, ¿permite lograr la creación de aprendizajes significativos?

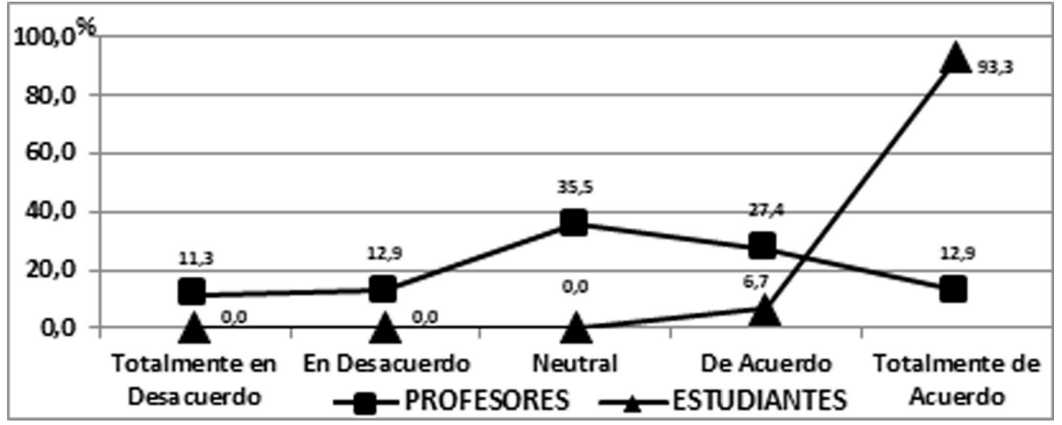

Fuente: Elaboración propia 
Profesores y estudiantes coinciden en concordar con la propuesta de la pregunta trabajo de campo y aprendizajes significativos. Sin embargo, el nivel de convencimiento de los estudiantes supera en 60 puntos a los profesores, estableciéndose una diferencia entre De Acuerdo y Totalmente de Acuerdo entre profesores (40,3\%) y estudiantes (100\%); la explicación de esta diferencia se ha propuesto en preguntas anteriores y dice relación con el ambiente laboral respecto del ambiente universitario.

\section{Síntesis de respuestas de los profesores}

Las actitudes de los profesores sobre la aplicación de métodos de la observación directa para enseñar geografía distan de ser unánimes; solo un $43,6 \%$ es consecuente con dichos métodos.

Consecuente con la respuesta anterior, es ampliamente compartido $(93,5 \%)$ que el trabajo de campo colabora con la comprensión de los fenómenos relativos al espacio geográfico.

Igualmente similar es la alta frecuencia $(95,1 \%)$ que opta por el trabajo de campo, en cuanto a que facilita una actitud crítica sobre entornos cercanos. Misma situación se presenta con una muy importante adhesión $(93,5 \%)$ al contacto con la realidad que promueve el desarrollo de valores; también concuerdan en la relación causal positiva entre observación directa y logro de aprendizajes significativos (95.1\%).

Finalmente, se valora la sistematicidad de las experiencias de terreno para aprender geografía, complementándose con la creatividad en el ámbito de los aprendizajes significativos.

\section{Síntesis de respuestas de los estudiantes}

Los estudiantes ratifican (70\%) su participación en actividades de terreno en la disciplina y de manera sistemática, según programa de estudios, en la Universidad de Los Lagos. Igualmente concuerdan $(86,6 \%)$ que el terreno debe ser planificado. El 95,1\% de los jóvenes se muestra partícipe en cuanto a vincular visita de terreno con adquisición de una actitud crítica respecto entorno. Muy asociado a lo anterior, estiman $(93,4 \%)$ las distintas vinculaciones con el medio como proactivas al desarrollo de valores. En ese ámbito, los aprendizajes son significativos en la opción de las experiencias previas, aspecto altamente apreciado (90\%).

En total acuerdo (100\%), los estudiantes concluyen que el trabajo al aire libre potencia los aprendizajes, significando así una ocasión integradora y coherente al conjunto de respuestas anteriores. 
Una convergencia importante (70\%) se expresa respecto de la sistematicidad en las oportunidades para enseñar y aprender al exterior del aula. En dicho ámbito se inscribe metodológicamente el trabajo de campo, en tanto facilitador de aprendizajes significativos.

Finalizado el análisis de la primera categoría clasificatoria, se despliega a continuación la segunda categoría denominada "observación directa".

\section{Categoría: Observación directa}

\section{Figura 10}

Pregunta 4: El contacto directo con el espacio geográfico, ¿es una estrategia de motivación para el aprendizaje en los alumnos/as?

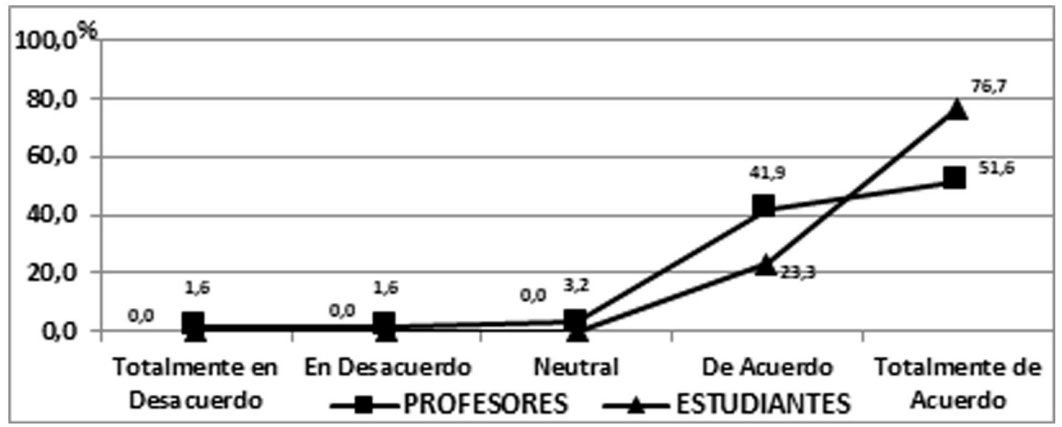

Fuente: Elaboración propia

Se observa una clara confluencia entre docentes y estudiantes, ya que sumando los indicadores De Acuerdo y Totalmente de Acuerdo el resultado es $93,5 \%$ profesores y $100 \%$ estudiantes. Se ratifica así que el trabajo educativo en exteriores motiva al discente y confirma la complejidad que reviste.

\section{Figura 11}

Pregunta 7: El trabajo al aire libre, ¿necesita de un estudiante con conocimientos básicos para analizar la realidad del entorno?

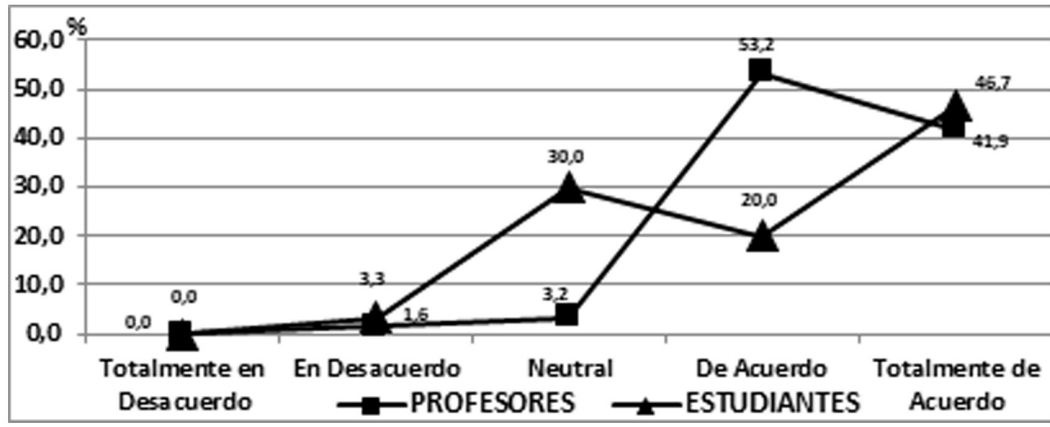

Fuente: Elaboración propia 
Si bien, la comparación entre De Acuerdo y Totalmente de Acuerdo de profesores y estudiantes es coincidente en cuanto a ratificar la necesidad de los conocimientos básicos previos, se produce una suerte de anomalía, considerando que la opinión de los docentes se eleva a un 95,1\% mientras que los estudiantes quedan 28,4 puntos porcentuales más bajo.

\section{Figura 12}

Pregunta 8: El trabajo de campo, ¿es una metodología que permite la sistematización de contenidos tratados en clase?

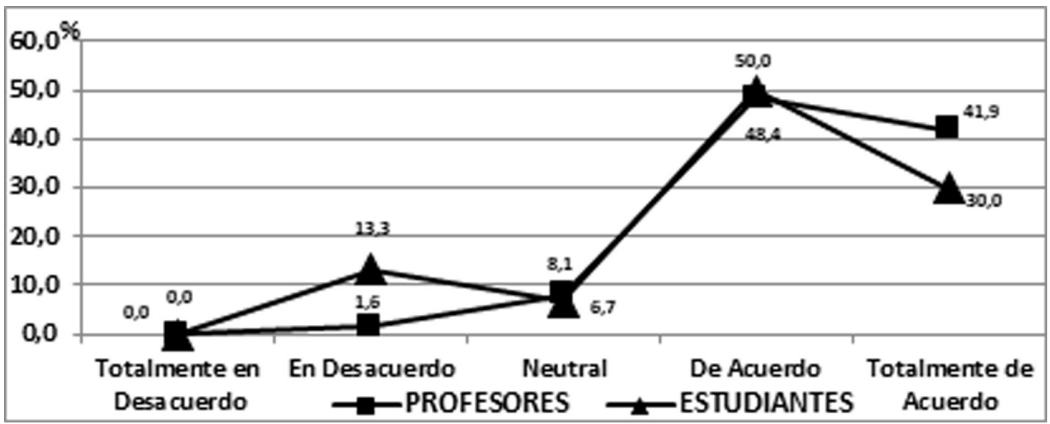

Fuente: Elaboración propia

En gran medida, estudiantes y profesores coinciden en opinar que el terreno aporta a nivel de interpretación explicativa del funcionamiento de los paisajes y su respectiva complejidad. La diferencia entre ambos grupos consultados radica en la importancia que los estudiantes le dan al En Desacuerdo y Neutral, totalizando un $20 \%$.

\section{Figura 13}

Pregunta 9: Cuando realiza trabajos de campo con sus estudiantes, ¿elabora usted guías de aprendizaje?

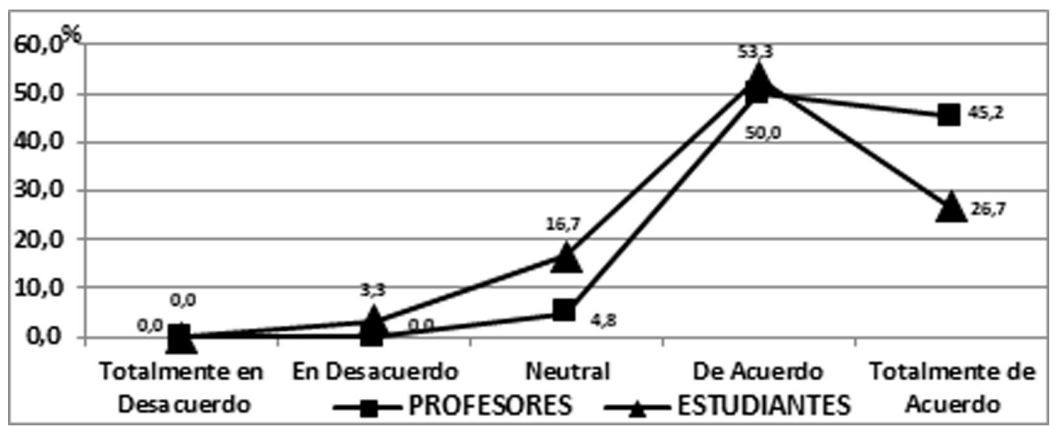

Fuente: Elaboración propia 
Mediante un alto porcentaje, mayor en los docentes, ambos actores ratifican ampliamente diseñar guías preestablecidas para trabajar en terreno. Sin embargo, los estudiantes se sitúan 15 puntos porcentuales por debajo de los profesores en su opinión De Acuerdo y Totalmente de Acuerdo: 80\% respecto de $95,2 \%$. En particular, dicha diferencia también se observa al comparar la opinión Totalmente de Acuerdo, que acumuló un 45,2\% en docentes y un $26,7 \%$ en estudiantes.

Figura 14

Pregunta 11: ¿Considera relevante el trabajo de campo como metodología didáctica?

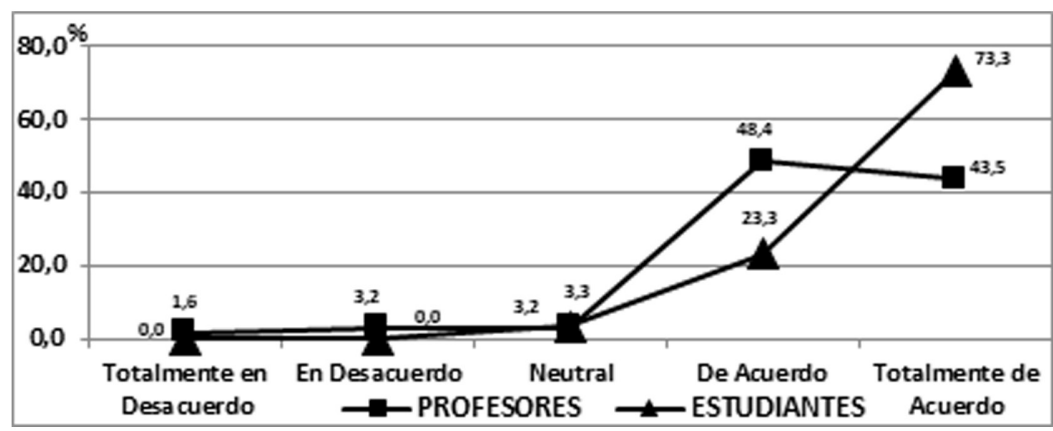

Fuente: Elaboración propia

Se observa una altísima coincidencia entre profesores y estudiantes respecto a validar el trabajo de campo como método de análisis; solo con cuatro puntos porcentuales de diferencia en De Acuerdo y Totalmente de Acuerdo.

Una diferencia sustantiva, sin embargo, se presenta en la opinión, un tanto extrema, de Totalmente de Acuerdo que muestra a los estudiantes con un $73,3 \%$, en tanto que los docentes apoyan dicha actitud con un $43,5 \%$.

Figura 15

Pregunta 14: ¿El trabajo de campo es desarrollado de forma interdisciplinar?

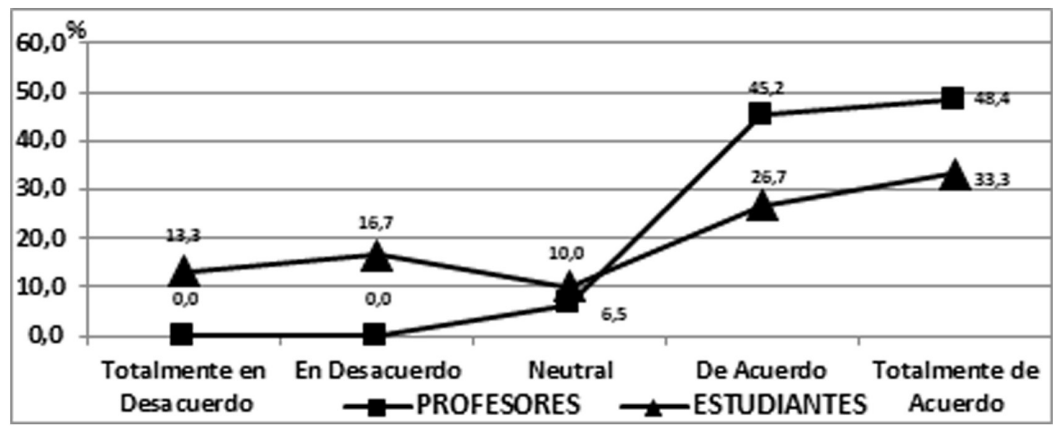

Fuente: Elaboración propia 
Ampliamente, los docentes (93,6\% en De Acuerdo y Totalmente de Acuerdo) demuestran más claridad, seguramente desarrollada porsu propia experiencia. Mientras que los estudiantes $(60 \%)$ no consideran necesario trabajar con docentes pertenecientes a más de una disciplina. Probablemente esto deba asumirse como una necesidad de incorporar en la formación el trabajo de interacción interdisciplinaria.

Figura 16

Pregunta 15: ¿Visita con anterioridad el lugar geográfico antes de desarrollar el trabajo de campo con sus estudiantes?

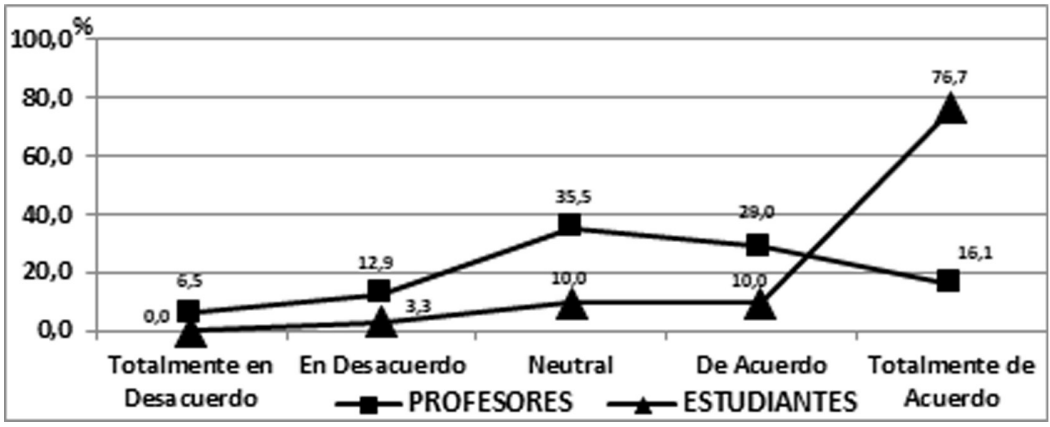

Fuente: Elaboración propia

Los estudiantes comprenden de mejor manera lo que implica la visita previa para optimizar la calidad del aprendizaje exclusivo que solo se puede lograr fuera del aula. Así lo demuestra la distancia porcentual de los niveles De Acuerdo y Totalmente de Acuerdo: 86,7\% estudiantes respecto a 45, 1\% profesores, una jornada laboral densa es radicalmente distinta a la vida académica del estudiante.

\section{Síntesis de respuestas de los profesores}

El estudiante necesita conocimientos básicos y conceptuales para analizar su entorno; es una condición que apoya muy ampliamente el $95.1 \%$ de los consultados. Este resultado valida que el trabajo de campo facilita la sistematización de aquellas materias trabajadas en la clase.

Que el estudiante disponga de una guía de trabajo para aprender geografía representa el 95,2\%; apreciar que un documento que oriente al grupocurso puede permitir registrar conocimientos básicos, sistematizar materias y testimoniar por qué convocar a más de una disciplina científica.

Se entiende que el análisis y comprensión de un espacio complejo convoca a más de una disciplina para interrogar los hechos geográficos, por lo tanto, 
es necesaria la interdisciplinariedad en el trabajo de campo, conformada por un $93,6 \%$.

El 91,9\% de los docentes asocia el trabajo de campo -método distintivo de la disciplina- como una eficiente estrategia para enseñar (aprender geografía en un contexto próximo a la transposición didáctica); alusión que se refiere a la adecuación o adaptación de determinados temas a materias científicas, para transformarlas a contenidos adaptados a niveles de madurez de determinados grupos escolares según la edad.

Las opciones se dispersan mostrando, presumiblemente, desorientación de los profesores a la hora de responder si concurren al lugar de trabajo con anterioridad a la visita con el curso: solo un 45,1\% lo hace. Genera todo un enigma aquel grupo numeroso de encuestados (35,5\%) que se refugia en Neutral; mientras que el 19,4\% se declara en Desacuerdo en complementar la planificación previa.

La motivación de salir y cambiar el escenario cotidiano del grupo-curso, es una situación que atrae la confirmación del 93,5\% de los docentes.

De modo general, se concluye que las actividades fuera del aula son valoradas por la mayoría de los docentes que participaron del cuestionario. Dicha tendencia sería más fructífera para las comunidades escolares y el interés y beneficio de equipos docentes, si se diseñara programas de perfeccionamiento con la participación de los beneficiarios: docentes y estudiantes; tal vez apoyados en líneas investigativas desarrolladas desde la Didáctica.

\section{Síntesis de respuestas de los estudiantes}

Para los estudiantes, disponer de conocimientos básicos para el análisis de terreno no es del todo necesario; solo el $66,7 \%$ concuerda con esa condición. No pronunciarse (Neutral) atrajo el tercio faltante. Un grupo muy mayoritario (80\%) acepta que el trabajo fuera del aula tiene la gran ventaja de sistematizar aquellos contenidos previamente tratados en aula. Por su parte, la interdisciplinariedad en terreno no resulta valorizada de modo suficiente (60\%). Considerando que dicha práctica no es prescindible, comporta un problema a resolver, atendiendo a la multicausalidad presente en los fenómenos socioespaciales.

La producción de guías para observar y registrar en terreno resulta muy exitosa $(80 \%)$, considerando que todos los estudiantes son proactivos a participar; aspecto que conduce a valorar al trabajo de campo -propio de la investigación geográfica-como metodología para un aprendizaje posibilitado por la transposición didáctica, y que es validado por $96,6 \%$ de los estudiantes. A través de una frecuencia menor a la recién mencionada $(86,7 \%)$, los 
jóvenes avalan una necesaria visita anterior para precisar adecuadamente ¿qué observarán? y ¿cómo y qué actividades realizarán?

Finalmente, el carácter motivador de observar a escala real es suscrito por todos los estudiantes que participaron en el cuestionario, cuestión que ratifica el logro de aprendizajes significativos.

\section{b) Entrevista}

Este instrumento consta de cinco preguntas abiertas y está orientado a conocer las opiniones de una manera más amplia y así complementar las respuestas del cuestionario. Fue aplicado a quince profesores y ocho estudiantes. A continuación se presentan las preguntas:

1. El trabajo de campo, como estrategia metodológica, ¿favorece la comprensión de procesos que ocurren sobre el espacio geográfico y desarrolla un sujeto consciente de su realidad? Fundamente.

2. ¿Por qué el contacto con la realidad favorece el desarrollo de valores?

3. Considera usted que, para realizar un trabajo de campo, deben poseer los conocimientos básicos ¿antes o después de la actividad realizada?

4. Cuando realiza un trabajo de campo, ¿responde una guía de aprendizaje preestablecida? SI - NO. Fundamente.

5. Al momento de organizar una salida a terreno, ¿aprovecha esa instancia para trabajar interdisciplinariamente? SI - NO. Fundamente.

\section{Síntesis de respuestas de los profesores}

Según los docentes, el trabajo de campo como estrategia metodológica es una forma de enseñanza significativa, ya que permite a los jóvenes aprender en terreno sobre la realidad histórica y geográfica de un lugar determinado, favorece además la comprensión dinámica y las consecuencias de los fenómenos sobre el medio natural.

A través de la interacción de lo práctico y teórico, los estudiantes comprenden mejor el medio geográfico y se sensibilizan sobre el comportamiento del ser humano y el impacto que este produce sobre el espacio.

El contacto con la realidad es una forma de desarrollar los valores en los estudiantes y de adquirir conductas acordes a su quehacer como individuos responsables de mantener el equilibrio entre los distintos elementos que conforman el espacio natural. 
Es relevante también destacar que seleccionar actividades con el grupocurso, al aire libre, les permite desarrollar: trabajo en equipo, un grado de impacto con la observación directa, valoración del medio que los rodea y un grado de concientización frente a problemas visibles. Vivenciar una realidad permite aprendizajes con sentido.

Para realizar trabajo de campo, afirman que debe haber una base teórica, por tanto, es responsabilidad del docente preparar al estudiante para lograr mejores resultados en sus aprendizajes. Contextualizar el contenido es fundamental, planificando la actividad con objetivos claros, tanto al inicio de la salida a terreno como posterior a ella.

Respecto al tipo de guía diseñada para utilizar con los estudiantes, los profesores señalan que entregan una guía preestablecida, pero dando los espacios para que el estudiante anote sus impresiones y realice intervenciones personales, expresando con libertad lo que les pareció interesante, haciendo comentarios y consultas.

En cuanto a aprovechar la instancia de una salida a terreno de forma interdisciplinaria con otros colegas, estos afirman que no es muy frecuente, por una situación administrativa, pero cuando tienen la posibilidad, esta se aprovecha, sobre todo en visitas a museos o parques nacionales.

\section{Síntesis de respuestas de los estudiantes}

En relación con la comprensión del espacio geográfico mediante el trabajo de campo y el desarrollo de un sujeto más consciente de su propia realidad, los estudiantes afirman que, en la medida que la actividad no solo sea descripción de elementos, sino también se dirija a la problematización y el análisis de lo observado, el objetivo se cumple.

Al contactar a un estudiante con la realidad, esta experiencia le permite ser un sujeto consciente y responsable de su entorno más cercano, generando un sentido de pertenencia, y tornándolo consciente de su realidad, apreciando de mejor manera el lugar que habita, aportando soluciones a problemas visibles. Los estudiantes, futuros pedagogos, están seguros de que las actividades al aire libre contribuyen a la formación y construcción de su identidad, además de la comprensión de procesos. En la medida en que el alumno participe del conocimiento de su realidad, se hace parte de él, se apropia de él y es más responsable.

El desarrollo de los valores a partir de las actividades al aire libre es un tema en que los ocho estudiantes entrevistados están de acuerdo, ya que crea nuevas relaciones y contacto con el grupo-curso. Aluden a que el desarrollo valórico se materializará al observar vertederos, playas contaminadas etc., produciendo rechazo sobre las medidas que no se aplican para revertir 
estas situaciones. Conocer realidades les genera empatía, respeto, cuidado, incluso sentirse parte de ella.

Respecto de los conocimientos previos que debe tener un alumno al momento de realizar una salida de campo, los estudiantes de la carrera de Pedagogía en Historia y Geografía afirman que sí es importante, sobre todo lo que dice relación con la ubicación espacial, lo que generaría un mejor análisis y reflexión del lugar que visitan. Afirman además que la base teórica es fundamental.

En cuanto al tipo de guías que reciben de parte de sus profesores en la universidad, al concretar una salida a terreno, responden que esta es preestablecida y sirve para ordenar información, pero al mismo tiempo dejan espacios para anotar impresiones generando una conciencia crítica de lo observado.

Consideran muy importante la interdisciplinariedad al momento de salir a un terreno, pero afirman también que no es común que esto suceda al interior de las asignaturas de Geografía, señalando que estas instancias no son aprovechadas.

\section{Conclusiones}

a. Área temática: experiencias con el trabajo de campo.

La aplicación de metodologías para el trabajo de terreno se explicita a través de dos preguntas en cada una de las dos Categorías.

Las respuestas de los docentes en la Categoría I, muestran una tendencia importante hacia el trabajo docente al interior del aula, mientras que las actividades en contacto con la realidad no exceden el tercio como estrategia metodológica.

En cuanto a la Categoría II, sobre observación directa, los profesores demuestran en su mayoría elaborar guías para observar y describir el espacio. Esta preparación de documentos para orientar la observación y las experiencias involucradas demuestra una actitud positiva, dado que favorece el logro de los objetivos a partir de la estrategia didáctica.

Los estudiantes (Universidad de Los Lagos), futuros docentes, con sus experiencias sistemáticas de terreno, se sitúan en una actitud crítica respecto del entorno y el desarrollo de valores que contribuyen a los aprendizajes significativos.

\section{b. Área temática: percepción de las experiencias en el trabajo de} terreno.

A diferencia del área temática anterior, basada en un total de cuatro preguntas que indagan sobre la participación efectiva en experiencias de terreno, esta área expone características relevantes de dicha actividad. 
Las respuestas permiten develar una actitud positiva según las ventajas metodológicas que representa el contacto con la realidad. Manifiestan que se promueve una percepción consciente de su realidad cercana y la comprensión de la diversidad de espacios ligados al aprecio hacia su entorno. Consideran los aprendizajes como significativos, apoyados en la motivación que produce la actividad.

Sin embargo, lo anterior permite constatar la ausencia de ideas clave asociadas al trabajo de campo, al menos en la dimensión teórico-conceptual. Es el caso, por ejemplo, del aprendizaje activo, promover la capacidad creativa para la construcción de nuevos conocimientos de parte de los estudiantes, y las experiencias previas como una de las condicionantes del constructivismo.

El presente trabajo plantea, de modo emergente, nuevas interrogantes que se deben ponderar como urgentes, no solo la de investigar, sino también la de intervenir. ¿Estamos todavía en esta materia más próximos a la situación que para los años 90 plantean las autoras Durán, Lara y Daguerre en 1993? A modo de hipótesis y provisionalmente, se propone que sí, porque efectivamente el trabajo de campo en el entorno real, orientado por una guía que propone y organiza experiencias, reviste una alta complejidad si se considera el principio de calidad como contexto. Se compromete un aprendizaje activo que tiene dos bases disciplinarias: las estrategias didácticas y el análisis espacial.

Se considera necesario incrementar los estudios locales para perfeccionar el método de trabajo y las capacidades científicas. Lo anterior contribuiría a que las comunidades educativas y las universidades formadoras de pedagogos en la especialidad pudieran enfrentar con mayor acierto las dificultades que hoy día afectan a la geografía en su importante contribución al ámbito de la didáctica.

\section{Referencias bibliográficas}

\section{Fuentes Primarias}

a) Documentos oficiales

Ministerio de Educación de Chile (1997). Objetivos Fundamentales y Contenidos Mínimos Obligatorios de la Educación Media. Consulta Nacional. Santiago: MINEDUC. 


\section{Fuentes Secundarias}

a) Artículos y capítulos de libros

Montilla, A. (2005). "El Trabajo de Campo: estrategia didáctica en la enseñanza de la geografía", en Geoenseñanza, Vol. 10, № 2, pp. 187-195.

Pérez, A. (2006). "La salida de campo: Una manera de enseñar y aprender Geografía", en Geoenseñanza, Vol. 11, № 2, pp. 22-234.

Sánchez, A. (1997). "La observación e interpretación del paisaje", en Didáctica Geográfica, № 2, pp. 45-56.

Santiago, R. J. (2008). "El contexto histórico: el cambio paradigmático y sus repercusiones en la enseñanza y el aprendizaje en la práctica escolar cotidiana", en Revista EDUCERE, Vol. 12, № 40, pp. 31-39.

Santiago, R. J. (2017). "La construcción del conocimiento en la enseñanza geográfica y la explicación de la realidad sociohistórica contemporánea", en Revista Paradigma, Vol. XXXVIII, № 2, pp. 140-159.

\section{b) Libros}

Araya, F. y M. González (2009). Presente y futuro de la Educación Geográfica en Chile. La Serena, Chile: Universidad de La Serena.

Bailey, P. (1983). Didáctica de la Geografía. Madrid: Cincel-Kapelusz.

Durán, D., A. Lara y C. Daguerre (1993). Los cambios mundiales y la enseñanza de la geografía. Buenos Aires: Troquel.

Giacobbe, M. (1998). Enseñar y aprender Ciencias Sociales: Tercer Ciclo EGB Polimodal. Buenos Aires: Homo Sapiens.

Hernández, R., C. Fernández, y I. Baptista (1998). Metodología de la Investigación. México: Mc Graw Hill ( $2^{\mathrm{a}}$ ed.).

Rodríguez, L. (2007). Una geografía escolar (in)visible. Bogotá: Universidad Distrital Francisco José de Caldas.

Souto, X. (1999). Didáctica de la Geografía. Problemas sociales y conocimiento del medio. Barcelona: Del Serbal.

Tatter, H. (1983). Didáctica de las Ciencias Sociales. Valdivia: Universidad Austral de Chile. 